\title{
Viscosity approximation methods for asymptotically nonexpansive mapping in CAT(0) spaces
}

Rabian Wangkeeree ${ }^{1,2^{*}}$, Uraiwan Boonkong ${ }^{1}$ and Pakkapon Preechasilp ${ }^{3}$

\section{${ }^{*}$ Correspondence:}

rabianw@nu.ac.th

1 Department of Mathematics,

Faculty of Science, Naresuan

University, Phitsanulok, 65000,

Thailand

${ }^{2}$ Centre of Excellence in

Mathematics, CHE, Si Ayutthaya Rd.,

Bangkok, 10400, Thailand

Full list of author information is

available at the end of the article

\begin{abstract}
Let $C$ be a nonempty, closed, and convex subset of a complete CAT(0) space $X$ and let $T$ be an asymptotically nonexpansive mapping of $C$ into itself such that the set of common fixed points of $T$ is nonempty. We introduce the iterative schemes for finding the common fixed point of an asymptotically nonexpansive mapping which is the unique solution of some variational inequalities. The strong convergence theorem of the proposed iterative schemes is established. Our result improves and generalizes several other results in the literature.
\end{abstract}

Keywords: viscosity approximation method; asymptotically nonexpansive mapping; variational inequality; CAT(0) space; common fixed point

\section{Introduction}

Let $(X, d)$ be a metric space. A geodesic path joining $x \in X$ to $y \in X$ (or, more briefly, a geodesic from $x$ to $y$ ) is a map $c$ from a closed interval $[0, l] \subset \mathbb{R}$ to $X$ such that $c(0)=x$, $c(l)=y$, and $d\left(c(t), c\left(t^{\prime}\right)\right)=\left|t-t^{\prime}\right|$ for all $t, t^{\prime} \in[0, l]$. In particular, $c$ is an isometry and $d(x, y)=l$. The image $\alpha$ of $c$ is called a geodesic (or metric) segment joining $x$ and $y$. When it is unique this geodesic segment is denoted by $[x, y]$. The space $(X, d)$ is said to be a geodesic space if every two points of $X$ are joined by a geodesic, and $X$ is said to be uniquely geodesic if there is exactly one geodesic joining $x$ and $y$ for each $x, y \in X$. A subset $Y \subseteq X$ is said to be convex if $Y$ includes every geodesic segment joining any two of its points. A geodesic triangle $\Delta\left(x_{1}, x_{2}, x_{3}\right)$ in a geodesic metric space $(X, d)$ consists of three points $x_{1}, x_{2}, x_{3}$ in $X$ (the vertices of $\triangle$ ) and a geodesic segment between each pair of vertices (the edges of $\triangle)$. A comparison triangle for the geodesic triangle $\Delta\left(x_{1}, x_{2}, x_{3}\right)$ in $(X, d)$ is a triangle $\bar{\triangle}\left(x_{1}, x_{2}, x_{3}\right):=\triangle\left(\bar{x}_{1}, \bar{x}_{2}, \bar{x}_{3}\right) \mathbb{E}^{2}$ such that $d_{\mathbb{E}_{2}}\left(\bar{x}_{i}, \bar{x}_{j}\right)=d\left(x_{i}, x_{j}\right)$ for all $i, j \in\{1,2,3\}$.

A geodesic space is said to be a $\mathrm{CAT}(0)$ space if all geodesic triangles of appropriate size satisfy the following comparison axiom.

$\mathrm{CAT}(0)$ : Let $\triangle$ be a geodesic triangle in $X$ and let $\bar{\Delta}$ be a comparison triangle for $\Delta$. Then $\triangle$ is said to satisfy the CAT(0) inequality if for all $x, y \in \Delta$ and all comparison points $\bar{x}, \bar{y} \in \bar{\Delta}$,

$$
d(x, y) \leq d_{\mathbb{E}^{2}}(\bar{x}, \bar{y})
$$

\section{Springer}

O 2015 Wangkeeree et al.; licensee Springer. This is an Open Access article distributed under the terms of the Creative Commons Attribution License (http://creativecommons.org/licenses/by/4.0), which permits unrestricted use, distribution, and reproduction in any medium, provided the original work is properly credited. 
If $x, y_{1}, y_{2}$ are points in a $\operatorname{CAT}(0)$ space and if $y_{0}$ is the midpoint of the segment $\left[y_{1}, y_{2}\right]$, then the $\operatorname{CAT}(0)$ inequality implies

$$
d^{2}\left(x, y_{0}\right) \leq \frac{1}{2} d^{2}\left(x, y_{1}\right)+\frac{1}{2} d^{2}\left(x, y_{2}\right)-\frac{1}{4} d^{2}\left(y_{1}, y_{2}\right) .
$$

This is the (CN)-inequality of Bruhat and Tits [1]. In fact ( $c f$. [2, p.163]), a geodesic space is a $\operatorname{CAT}(0)$ space if and only if it satisfies the $(\mathrm{CN})$-inequality.

It is well known that any complete, simply connected Riemannian manifold having nonpositive sectional curvature is a CAT (0) space. Other examples include Pre-Hilbert spaces, $\mathbb{R}$-trees (see [2]), Euclidean buildings (see [3]), the complex Hilbert ball with a hyperbolic metric (see [4]), and many others. Complete CAT(0) spaces are often called Hadamard spaces. It is proved in [2] that a normed linear space satisfies the $(\mathrm{CN})$-inequality if and only if it satisfies the parallelogram identity, i.e., it is a pre-Hilbert space; hence it is not so unusual to have an inner product-like notion in Hadamard spaces. Berg and Nikolaev [5] introduced the concept of quasilinearization as follows:

Let us formally denote a pair $(a, b) \in X \times X$ by $\overrightarrow{a b}$ and call it a vector. Then quasilinearization is defined as a map $\langle\cdot, \cdot\rangle:(X \times X) \times(X \times X) \rightarrow \mathbb{R}$ defined by

$$
\langle\overrightarrow{a b}, \overrightarrow{c d}\rangle=\frac{1}{2}\left(d^{2}(a, d)+d^{2}(b, c)-d^{2}(a, c)-d^{2}(b, d)\right) \quad(a, b, c, d \in X)
$$

It is easily seen that $\langle\overrightarrow{a b}, \overrightarrow{c d}\rangle=\langle\overrightarrow{c d}, \overrightarrow{a b}\rangle,\langle\overrightarrow{a b}, \overrightarrow{c d}\rangle=-\langle\overrightarrow{b a}, \overrightarrow{c d}\rangle$ and $\langle\overrightarrow{a x}, \overrightarrow{c d}\rangle+\langle\overrightarrow{x b}, \overrightarrow{c d}\rangle=\langle\overrightarrow{a b}, \overrightarrow{c d}\rangle$ for all $a, b, c, d, x \in X$. We say that $X$ satisfies the Cauchy-Schwarz inequality if

$$
\langle\overrightarrow{a b}, \overrightarrow{c d}\rangle \leq d(a, b) d(c, d)
$$

for all $a, b, c, d \in X$. It known [5, Corollary 3] that a geodesically connected metric space is a CAT(0) space if and only if it satisfies the Cauchy-Schwarz inequality.

In 2010, Kakavandi and Amini [6] introduced the concept of dual space for $\mathrm{CAT}(0)$ spaces as follows. Consider the map $\Theta: \mathbb{R} \times X \times X \rightarrow C(X)$ defined by

$$
\Theta(t, a, b)(x)=t\langle\overrightarrow{a b}, \overrightarrow{a x}\rangle,
$$

where $C(X)$ is the space of all continuous real-valued functions on $X$. Then the CauchySchwarz inequality implies that $\Theta(t, a, b)$ is a Lipschitz function with Lipschitz semi-norm $L(\Theta(t, a, b))=|t| d(a, b)$ for all $t \in \mathbb{R}$ and $a, b \in X$, where

$$
L(f)=\sup \left\{\frac{f(x)-f(y)}{d(x, y)}: x, y \in X, x \neq y\right\}
$$

is the Lipschitz semi-norm of the function $f: X \rightarrow \mathbb{R}$. Now, define the pseudometric $D$ on $\mathbb{R} \times X \times X$ by

$$
D((t, a, b),(s, c, d))=L(\Theta(t, a, b)-\Theta(s, c, d)) .
$$

Lemma 1.1 [6, Lemma 2.1] $D((t, a, b),(s, c, d))=0$ if and only if $t\langle\overrightarrow{a b}, \overrightarrow{x y}\rangle=s\langle\overrightarrow{c d}, \overrightarrow{x y}\rangle$ for all $x, y \in X$. 
For a complete $\operatorname{CAT}(0)$ space $(X, d)$, the pseudometric space $(\mathbb{R} \times X \times X, D)$ can be considered as a subspace of the pseudometric space $(\operatorname{Lip}(X, R), L)$ of all real-valued Lipschitz functions. Also, $D$ defines an equivalence relation on $\mathbb{R} \times X \times X$, where the equivalence class of $t \overrightarrow{a b}:=(t, a, b)$ is

$$
[t \overrightarrow{a b}]=\{\overrightarrow{s c d}: t\langle\overrightarrow{a b}, \overrightarrow{x y}\rangle=s\langle\overrightarrow{c d}, \overrightarrow{x y}\rangle \forall x, y \in X\}
$$

The set $X^{*}:=\{[\overrightarrow{t a b}]:(t, a, b) \in \mathbb{R} \times X \times X\}$ is a metric space with metric $D$, which is called the dual metric space of $(X, d)$. Recently, Dehghan and Rooin [7] introduced the duality mapping in $\mathrm{CAT}(0)$ spaces and studied its relation with subdifferential, by using the concept of quasilinearization. Then they presented a characterization of metric projection in CAT(0) spaces as follows.

Theorem 1.2 [7, Theorem 2.4] Let $C$ be a nonempty convex subset of a complete $C A T(0)$ space $X, x \in X$ and $u \in C$. Then

$$
u=P_{C} x \quad \text { if and only if } \quad\langle\overrightarrow{y u}, \overrightarrow{u x}\rangle \geq 0, \quad \text { for all } y \in C
$$

Let $C$ be a nonempty subset of a complete CAT(0) space $X$. Then a mapping $T$ of $C$ into itself is called nonexpansive iff $d(T x, T y) \leq d(x, y)$ for all $x, y \in C$. A point $x \in C$ is called a fixed point of $T$ if $x=T x$. We denote by $F(T)$ the set of all fixed points of $T$. Kirk [8] showed that the fixed point set of a nonexpansive mapping $T$ is closed and convex. A mapping $T$ of $C$ into itself is called asymptotically nonexpansive if there exists a sequence $\left\{k_{n}\right\} \subset[1, \infty)$ with $k_{n} \rightarrow 1$ such that $d\left(T^{n} x, T^{n} y\right) \leq k_{n} d(x, y)$ for all integers $n \geq 1$ and all $x, y \in C$. A mapping $f$ of $C$ into itself is called a contraction with coefficient $\alpha \in(0,1)$ iff $d(f(x), f(y)) \leq \alpha d(x, y)$ for all $x, y \in C$. Banach's contraction principle [9] guarantees that $f$ has a unique fixed point when $C$ is a nonempty, closed, and convex subset of a complete metric space. The existence of fixed points and convergence theorems for several mappings in CAT(0) spaces has been investigated by many authors (see also [10-15]).

Clearly, every contraction mapping is nonexpansive and every nonexpansive mapping is asymptotically nonexpansive with sequence $k_{n}=1$, for all $n \geq 1$. However, asymptotically nonexpansive mappings which are not nonexpansive (see, e.g., [16]). As a generalization of the class of nonexpansive mappings, the class of asymptotically nonexpansive mappings was introduced by Goebel and Kirk [16]) in 1972 and has been studied by several authors. Goebel and Kirk proved that if $C$ is a nonempty, closed, convex, and bounded subset of a uniformly convex Banach space (more general than a Hilbert space, i.e., CAT(0) space), then every asymptotically nonexpansive self-mapping of $C$ has a fixed point. The weak and strong convergence problems to fixed points of nonexpansive and asymptotically nonexpansive mappings have been studied by many authors.

One classical way to study nonexpansive mappings is to use contractions to approximate nonexpansive mappings. More precisely, take $t \in(0,1)$ and define a contraction $T_{t}: C \rightarrow C$ by

$$
T_{t}=t u+(1-t) T x, \quad \forall x \in C,
$$

where $u \in C$ is an arbitrary fixed element. Banach's contraction mapping principle guarantees that $T_{t}$ has a unique fixed point $x_{t}$ in $C$. It is unclear, in general, what the behavior 
of $x_{t}$ is as $t \rightarrow 0$, even if $T$ has a fixed point. However, in the case of $T$ having a fixed point, Browder [17] proved that $x_{t}$ converges strongly to a fixed point of $T$ that is nearest to $u$ in the frame work of Hilbert spaces. Reich [18] extended Browder's result to the setting of Banach spaces and proved, in a uniformly smooth Banach space, that $x_{t}$ converges strongly to a fixed point of $T$ and the limit defines the (unique) sunny nonexpansive retraction from $C$ onto $F(T)$. Halpern [19] introduced the following explicit iterative scheme (5) for a nonexpansive mapping $T$ on a subset $C$ of a Hilbert space by taking any points $u, x_{1} \in C$, defining the iterative sequence $\left\{x_{n}\right\}$ :

$$
x_{n+1}=\alpha_{n} u+\left(1-\alpha_{n}\right) T x_{n} .
$$

He proved that the sequence $\left\{x_{n}\right\}$ generated by (5) converges to a fixed point of $T$. In 2010, Saejung [15] studied the convergence theorems of the following Halpern iterations for a nonexpansive mapping $T$ in a complete CAT(0) space: Let $u$ be fixed and $x_{t} \in C$ be the unique fixed point of the contraction $x \mapsto t u \oplus(1-t) T x$; i.e.

$$
x_{t}=t u \oplus(1-t) T x t
$$

where $t \in[0,1], x_{1}, u \in C$ are arbitrary chosen, and

$$
x_{n+1}=\alpha_{n} u \oplus\left(1-\alpha_{n}\right) T x_{n}, \quad n \geq 1,
$$

where $\left\{\alpha_{n}\right\} \rightarrow(0,1)$. It is proved in [15] that $\left\{x_{t}\right\}$ converges strongly as $t \rightarrow 0$ to $\tilde{x} \in F(T)$ which is nearest to $\mathrm{u}\left(\tilde{x}=P_{F(T)} u\right)$ and $\left\{x_{n}\right\}$ converges strongly as $n \rightarrow \infty$ to $\tilde{x} \in F(T)$ which is nearest to $u$ under certain appropriate conditions on $\left\{\alpha_{n}\right\}$, where $P_{C} x$ is a metric projection from $X$ onto $C$. Moreover, the author applied his result to find a common fixed point of a countable family of nonexpansive mappings $\left\{T_{n}\right\}_{n=1}^{\infty}$. He proved that the following iterative scheme (8) converges strongly to $\tilde{x} \in \bigcap_{n=1}^{\infty} F\left(T_{n}\right)$ which is nearest to $u$ :

$$
x_{n+1}=\alpha_{n} u \oplus\left(1-\alpha_{n}\right) T_{n} x_{n}, \quad n \geq 1 .
$$

In 2012, Shi and Chen [14], studied the convergence theorems of the following Moudafi's viscosity iterations for a nonexpansive mapping $T$ : for a contraction $f$ on $C$ and $t \in(0,1)$, let $x_{t} \in C$ be the unique fixed point of the contraction $x \mapsto t f(x) \oplus(1-t) T x$; i.e.

$$
x_{t}=t f\left(x_{t}\right) \oplus(1-t) T x_{t},
$$

and $x_{1} \in C$ is arbitrary chosen and

$$
x_{n+1}=\alpha_{n} f\left(x_{n}\right) \oplus\left(1-\alpha_{n}\right) T x_{n}, \quad \forall n \geq 1,
$$

where $\left\{\alpha_{n}\right\} \subset(0,1)$. They proved that $\left\{x_{t}\right\}$ defined by (9) converges strongly as $t \rightarrow 0$ to $\tilde{x} \in$ $F(T)$ such that $\tilde{x}=P_{F(T)} f(\tilde{x})$ in the framework of CAT(0) space satisfying the property $\mathcal{P}$, i.e., if for $x, u, y_{1}, y_{2} \in X$,

$$
d\left(x, P_{\left[x, y_{1}\right]} u\right) d\left(x, y_{1}\right) \leq d\left(x, P_{\left[x, y_{2}\right]} u\right) d\left(x, y_{2}\right)+d(x, u) d\left(y_{1}, y_{2}\right) .
$$


Furthermore, they also found that $\left\{x_{n}\right\}$ defined by (10) converges strongly as $n \rightarrow \infty$ to $\tilde{x} \in F(T)$ under certain appropriate conditions imposed on $\left\{\alpha_{n}\right\}$. Recently, using the concept of quasilinearization, Wangkeeree and Preechasilp [20] studied the strong convergence theorems of the iterative schemes (9) and (10) in CAT(0) spaces without the property $\mathcal{P}$. They proved the iterative schemes (9) and (10) converge strongly to $\tilde{x}$ such that $\tilde{x}=P_{F(T)} f(\tilde{x})$ which is the unique solution of the variational inequality (VIP):

$$
\langle\overrightarrow{\tilde{x} f \tilde{x}}, \overrightarrow{x \tilde{x}}\rangle \geq 0, \quad x \in F(T)
$$

On the other hand, Shi et al. [21] studied the $\Delta$-convergence of the iteration sequence for asymptotically nonexpansive mappings in CAT(0) spaces. For related work, see [22-25].

Motivated and inspired by Saejung [15] and Wangkeeree and Preechasilp [20] and Shi et al. [21], the purpose of this paper is to study the strong convergence theorems of the Moudafi's viscosity approximation methods for an asymptotically nonexpansive mapping in CAT(0) spaces. Let $C$ be a closed convex subset of a complete CAT(0) space $X$. Let $T^{n}$ : $C \rightarrow C$ be an asymptotically nonexpansive mapping. For given a contraction $f$ on $C$ and $\alpha_{n} \in(0,1)$, let $x_{n} \in C$ be the unique fixed point of the contraction $x \mapsto \alpha_{n} f(x) \oplus\left(1-\alpha_{n}\right) T^{n} x$; i.e.

$$
x_{n}=\alpha_{n} f\left(x_{n}\right) \oplus\left(1-\alpha_{n}\right) T^{n} x_{n}, \quad n \geq 1,
$$

and $x_{1} \in C$ is arbitrary chosen and

$$
x_{n+1}=\alpha_{n} f\left(x_{n}\right) \oplus\left(1-\alpha_{n}\right) T^{n} x_{n}, \quad n \geq 1 .
$$

We prove the iterative schemes $\left\{x_{n}\right\}$ defined by (12) and (13) converge strongly to the same point $\tilde{x}$ such that $\tilde{x}=P_{\mathcal{F}} f(\tilde{x})$ which is the unique solution of the variational inequality:

$$
|\overrightarrow{\tilde{x} f(\tilde{x})}, \vec{x} \tilde{x}\rangle \geq 0, \quad x \in F(T)
$$

\section{Preliminaries}

In this paper, we write $(1-t) x \oplus t y$ for the unique point $z$ in the geodesic segment joining from $x$ to $y$ such that

$$
d(z, x)=t d(x, y), \quad \text { and } \quad d(z, y)=(1-t) d(x, y) .
$$

We also denote by $[x, y]$ the geodesic segment joining from $x$ to $y$, that is, $[x, y]=\{(1-t) x \oplus$ ty: $t \in[0,1]\}$. A subset $C$ of a CAT(0) space is convex if $[x, y] \subseteq C$ for all $x, y \in C$.

The following lemmas play an important role in our paper.

Lemma 2.1 [2, Proposition 2.2] Let $X$ be a $C A T(0)$ space, $p, q, r, s \in X$ and $\lambda \in[0,1]$. Then

$$
d(\lambda p \oplus(1-\lambda) q, \lambda r \oplus(1-\lambda) s) \leq \lambda d(p, r)+(1-\lambda) d(q, s) .
$$

Lemma 2.2 [11, Lemma 2.4] Let $X$ be a $C A T(0)$ space, $x, y, z \in X$ and $\lambda \in[0,1]$. Then

$$
d(\lambda x \oplus(1-\lambda) y, z) \leq \lambda d(x, z)+(1-\lambda) d(y, z) .
$$


Lemma 2.3 [11, Lemma 2.5] Let $X$ be a $C A T(0)$ space, $x, y, z \in X$ and $\lambda \in[0,1]$. Then

$$
d^{2}(\lambda x \oplus(1-\lambda) y, z) \leq \lambda d^{2}(x, z)+(1-\lambda) d^{2}(y, z)-\lambda(1-\lambda) d^{2}(x, y)
$$

The concept of $\Delta$-convergence introduced by Lim [26] in 1976 was shown by Kirk and Panyanak [27] in CAT(0) spaces to be very similar to the weak convergence in a Banach space setting. Next, we give the concept of $\Delta$-convergence and collect some basic properties.

Let $\left\{x_{n}\right\}$ be a bounded sequence in a $\operatorname{CAT}(0)$ space $X$. For $x \in X$, we set

$$
r\left(x,\left\{x_{n}\right\}\right)=\limsup _{n \rightarrow \infty} d\left(x, x_{n}\right) .
$$

The asymptotic radius $r\left(\left\{x_{n}\right\}\right)$ of $\left\{x_{n}\right\}$ is given by

$$
r\left(\left\{x_{n}\right\}\right)=\inf \left\{r\left(x,\left\{x_{n}\right\}\right): x \in X\right\},
$$

and the asymptotic center $A\left(\left\{x_{n}\right\}\right)$ of $\left\{x_{n}\right\}$ is the set

$$
A\left(\left\{x_{n}\right\}\right)=\left\{x \in X: r\left(x,\left\{x_{n}\right\}\right)=r\left(\left\{x_{n}\right\}\right)\right\} .
$$

It is well known from Proposition 7 of [13] that in a complete CAT(0) space, $A\left(\left\{x_{n}\right\}\right)$ consists of exactly one point. A sequence $\left\{x_{n}\right\} \subset X$ is said to $\Delta$-converge to $x \in X$ if $A\left(\left\{x_{n_{k}}\right\}\right)=\{x\}$ for every subsequence $\left\{x_{n_{k}}\right\}$ of $\left\{x_{n}\right\}$. Uniqueness of the asymptotic center implies that CAT(0) space $X$ satisfies Opial's property, i.e., for given $\left\{x_{n}\right\} \subset X$ such that $\left\{x_{n}\right\} \Delta$-converges to $x$ and given $y \in X$ with $y \neq x$,

$$
\limsup _{n \rightarrow \infty} d\left(x_{n}, x\right)<\limsup _{n \rightarrow \infty} d\left(x_{n}, y\right) \text {. }
$$

Since it is not possible to formulate the concept of demiclosedness in a CAT( 0$)$ setting, as stated in linear spaces, let us formally say that ' $I-T$ is demiclosed at zero' if the conditions, $\left\{x_{n}\right\} \subseteq C \Delta$-converges to $x$ and $d\left(x_{n}, T x_{n}\right) \rightarrow 0$ imply $x \in F(T)$.

Lemma 2.4 [27] Every bounded sequence in a complete CAT(0) space always has a $\Delta$ convergent subsequence.

Lemma 2.5 [12] If $C$ is a closed convex subset of a complete $C A T(0)$ space and if $\left\{x_{n}\right\}$ is a bounded sequence in $C$, then the asymptotic center of $\left\{x_{n}\right\}$ is in $C$.

Lemma 2.6 [28] If $C$ is a closed convex subset of $X$ and $T: C \rightarrow X$ is an asymptotically nonexpansive mapping, then the conditions $\left\{x_{n}\right\} \Delta$-convergence to $x$ and $d\left(x_{n}, T x_{n}\right) \rightarrow 0$, and imply $x \in C$ and $x \in F(T)$.

Having the notion of quasilinearization, Kakavandi and Amini [6] introduced the following notion of convergence.

A sequence $\left\{x_{n}\right\}$ in the complete $\operatorname{CAT}(0)$ space $(X, d) w$-converges to $x \in X$ if

$$
\lim _{n \rightarrow \infty}\left\langle\overrightarrow{x x}_{n}, \overrightarrow{x y}\right\rangle=0
$$

i.e. $\lim _{n \rightarrow \infty}\left(d^{2}\left(x_{n}, x\right)-d^{2}\left(x_{n}, y\right)+d^{2}(x, y)\right)=0$ for all $y \in X$. 
It is obvious that convergence in the metric implies $w$-convergence, and it is easy to check that $w$-convergence implies $\Delta$-convergence [6, Proposition 2.5], but it is showed in [29, Example 4.7] that the converse is not valid. However, the following lemma shows another characterization of $\Delta$-convergence as well as, more explicitly, a relation between $w$-convergence and $\Delta$-convergence.

Lemma 2.7 [29, Theorem 2.6] Let $X$ be a complete $C A T(0)$ space, $\left\{x_{n}\right\}$ be a sequence in $X$ and $x \in X$. Then $\left\{x_{n}\right\} \Delta$-converges to $x$ if and only if $\lim \sup _{n \rightarrow \infty}\left\langle\overrightarrow{x_{n}}, \overrightarrow{x y}\right\rangle \leq 0$ for all $y \in X$.

Lemma 2.8 [30, Lemma 2.1] Let $\left\{a_{n}\right\}$ be a sequence of non-negative real numbers satisfying the property

$$
a_{n+1} \leq\left(1-\alpha_{n}\right) a_{n}+\alpha_{n} \beta_{n}, \quad n \geq 0,
$$

where $\left\{\alpha_{n}\right\} \subseteq(0,1)$ and $\left\{\beta_{n}\right\} \subseteq \mathbb{R}$ such that

(i) $\sum_{n=0}^{\infty} \alpha_{n}=\infty$;

(ii) $\lim \sup _{n \rightarrow \infty} \beta_{n} \leq 0$ or $\sum_{n=0}^{\infty}\left|\alpha_{n} \beta_{n}\right|<\infty$.

Then $\left\{a_{n}\right\}$ converges to zero, as $n \rightarrow \infty$.

The following two vital lemmas can be found in [20].

Lemma 2.9 [20] Let $X$ be a complete $C A T(0)$ space. Then for all $u, x, y \in X$, the following inequality holds

$$
d^{2}(x, u) \leq d^{2}(y, u)+2\langle\overrightarrow{x y}, \overrightarrow{x u}\rangle .
$$

Lemma 2.10 [20] Let $X$ be a CAT(0) space. For any $t \in[0,1]$ and $u, v \in X$, let $u_{t}=t u \oplus$ $(1-t) v$. Then, for all $x, y \in X$,

(i) $\left\langle\overrightarrow{u_{t} x}, \overrightarrow{u_{t} y}\right\rangle \leq t\left\langle\overrightarrow{u_{x}}, \overrightarrow{u_{t} y}\right\rangle+(1-t)\left\langle\overrightarrow{v x}, \overrightarrow{u_{t} y}\right\rangle$;

(ii) $\left\langle\overrightarrow{u_{t} x}, \overrightarrow{u y}\right\rangle \leq t\langle\overrightarrow{u x}, \overrightarrow{u y}\rangle+(1-t)\langle\overrightarrow{v x}, \overrightarrow{u y}\rangle$ and $\left\langle\overrightarrow{u_{t} x}, \overrightarrow{v y}\right\rangle \leq t\langle\overrightarrow{u x}, \overrightarrow{v y}\rangle+(1-t)\langle\overrightarrow{v x}, \overrightarrow{v y}\rangle$.

\section{Viscosity approximation methods}

In this section, we present the strong convergence theorems of the Moudafi's viscosity approximation methods for asymptotically nonexpansive mapping $T: C \rightarrow C$ in a complete CAT(0) space.

Theorem 3.1 Let $C$ be a closed convex subset of a complete $C A T(0)$ space $X$, and let $T$ : $C \rightarrow C$ be an asymptotically nonexpansive mapping with a sequence $\left\{k_{n}\right\} \subset[1,+\infty)$ and $\lim _{n \rightarrow \infty} k_{n}=1$ such that $F(T) \neq \emptyset$. Let $f$ be a contraction on $C$ with coefficient $0<\alpha<1$. Let $\left\{\alpha_{n}\right\}$ be a sequence of real numbers with $0<\alpha_{n}<1$. Then the following statements hold:

(i) For each $n \in \mathbb{N}$, if $\frac{k_{n}-1}{\alpha_{n}}<1-\alpha$, then there exists $y_{n}$ such that

$$
y_{n}=\alpha_{n} f\left(y_{n}\right) \oplus\left(1-\alpha_{n}\right) T^{n} y_{n} .
$$

(ii) If $\alpha_{n} \rightarrow 0$ and $\frac{k_{n}-1}{\alpha_{n}} \rightarrow 0$ as $n \rightarrow \infty$, then $\left\{y_{n}\right\}$ converges strongly as $n \rightarrow \infty$ to $\tilde{x}$ such that $\tilde{x}=P_{F(T)} f(\tilde{x})$, which is equivalent to the following variational inequality:

$$
|\overrightarrow{\tilde{x} f(\tilde{x})}, \overrightarrow{x \tilde{x}}\rangle \geq 0, \quad x \in F(T) .
$$


Proof For each integer $n \leq 1$, define a mapping $G_{n}: C \rightarrow C$ by

$$
G_{n}(x)=\alpha_{n} f(x) \oplus\left(1-\alpha_{n}\right) T^{n} x, \quad \forall x \in C .
$$

We shall show that $G_{n}$ is a contraction mapping. For any $x, y \in C$

$$
\begin{aligned}
d\left(G_{n}(x), G_{n}(y)\right) & =d\left(\alpha_{n} f(x) \oplus\left(1-\alpha_{n}\right) T^{n} x, \alpha_{n} f(y) \oplus\left(1-\alpha_{n}\right) T^{n} y\right) \\
& \leq \alpha_{n} d(f(x), f(y))+\left(1-\alpha_{n}\right) d\left(T^{n} x, T^{n} y\right) \\
& \leq \alpha_{n} \alpha d(x, y)+\left(1-\alpha_{n}\right) k_{n} d(x, y) \\
& =\left(k_{n}-\alpha_{n} k_{n}+\alpha \alpha_{n}\right) d(x, y) .
\end{aligned}
$$

Since $0<\frac{k_{n}-1}{\alpha_{n}}<1-\alpha$, we have

$$
0<\frac{k_{n}-1}{\alpha_{n}}<1-\alpha \leq \alpha_{n} k_{n}-\alpha \alpha_{n} .
$$

It follows that $0<k_{n}-\alpha_{n} k_{n}+\alpha_{n} \alpha<1$. We see that $G_{n}$ is a contraction map with coefficient $\left(k_{n}-\alpha_{n} k_{n}+\alpha_{n} \alpha\right)$. For each integer $n \leq 1$, there exists a unique $y_{n} \in C$ such that $G_{n}\left(y_{n}\right)=y_{n}$, that is,

$$
y_{n}=\alpha_{n} f\left(y_{n}\right) \oplus\left(1-\alpha_{n}\right) T^{n} y_{n} .
$$

Next, we show that $\left\{y_{n}\right\}$ is bounded. For any $p \in F(T)$, we have

$$
\begin{aligned}
d\left(y_{n}, p\right) & =d\left(\alpha_{n} f\left(y_{n}\right) \oplus\left(1-\alpha_{n}\right) T^{n} y_{n}, p\right) \\
& \leq \alpha_{n} d\left(f\left(y_{n}\right), f(p)\right)+\alpha_{n} d(f(p), p)+\left(1-\alpha_{n}\right) d\left(T^{n} y_{n}, p\right) \\
& \leq \alpha \alpha_{n} d\left(y_{n}, p\right)+\alpha_{n} d(f(p), p)+k_{n}\left(1-\alpha_{n}\right) d\left(y_{n}, p\right) \\
& =\left\{k_{n}-\left(k_{n}-\alpha\right) \alpha_{n}\right\} d\left(y_{n}, p\right)+\alpha_{n} d(f(p), p) .
\end{aligned}
$$

Then

$$
d\left(y_{n}, p\right) \leq \frac{\alpha_{n}}{\left(k_{n}-\alpha\right) \alpha_{n}-\left(k_{n}-1\right)} d(f(p), p) \leq \frac{1}{1-\alpha} d(f(p), p) .
$$

Hence $\left\{y_{n}\right\}$ is bounded, and so are $\left\{T y_{n}\right\}$ and $\left\{f\left(y_{n}\right)\right\}$. We get

$$
\begin{aligned}
\lim _{n \rightarrow \infty} d\left(y_{n}, T^{n} y_{n}\right) & =\lim _{n \rightarrow \infty} d\left(\alpha_{n} f\left(y_{n}\right) \oplus\left(1-\alpha_{n}\right) T^{n} y_{n}, T^{n} y_{n}\right) \\
& \leq \lim _{n \rightarrow \infty}\left[\alpha_{n} d\left(f\left(y_{n}\right), T^{n} y_{n}\right)+\left(1-\alpha_{n}\right) d\left(T^{n} y_{n}, T^{n} y_{n}\right)\right] \\
& \leq \lim _{n \rightarrow \infty} \alpha_{n} d\left(f\left(y_{n}\right), T^{n} y_{n}\right) .
\end{aligned}
$$

Thus

$$
\lim _{n \rightarrow \infty} d\left(y_{n}, T^{n} y_{n}\right)=0
$$

Let $L=\sup _{n} k_{n}$, then we have

$$
d\left(T^{n} y_{n}, p\right) \leq k_{n} d\left(y_{n}, p\right) \leq L d\left(y_{n}, p\right) .
$$


It follows that the sequence $\left\{T^{n} y_{n}\right\}$ is bounded. We claim that $\lim _{n \rightarrow \infty} d\left(y_{n}, T y_{n}\right)=0$. Indeed, we have

$$
\begin{gathered}
d\left(y_{n}, y_{n-1}\right)=d\left(\alpha_{n} f\left(y_{n}\right) \oplus\left(1-\alpha_{n}\right) T^{n} y_{n}, y_{n-1}\right) \\
\leq\left(1-\alpha_{n}\right) d\left(T^{n} y_{n}, y_{n-1}\right) \\
\leq\left(1-\alpha_{n}\right) d\left(T^{n} y_{n}, y_{n}\right)+\left(1-\alpha_{n}\right) d\left(y_{n}, y_{n-1}\right) \\
\leq \frac{\left(1-\alpha_{n}\right)}{\alpha_{n}} d\left(T^{n} y_{n}, y_{n}\right) \rightarrow 0, \\
d\left(y_{n-1}, T^{n-1} y_{n}\right)=d\left(\alpha_{n-1} f\left(y_{n-1}\right) \oplus\left(1-\alpha_{n-1}\right) T^{n-1} y_{n-1}, T^{n-1} y_{n}\right) \\
\leq \alpha_{n-1} d\left(f\left(y_{n-1}\right), T^{n-1} y_{n}\right)+\left(1-\alpha_{n-1}\right) d\left(T^{n-1} y_{n-1}, T^{n-1} y_{n}\right) \\
\quad \leq\left(1-\alpha_{n-1}\right) L d\left(y_{n-1}, y_{n}\right) \rightarrow 0, \\
d\left(y_{n}, T^{n-1} y_{n}\right) \leq d\left(y_{n}, y_{n-1}\right)+d\left(y_{n-1}, T^{n-1} y_{n}\right) \rightarrow 0 .
\end{gathered}
$$

Thus

$$
\begin{aligned}
d\left(y_{n}, T y_{n}\right) & \leq d\left(y_{n}, T^{n} y_{n}\right)+d\left(T^{n} y_{n}, T y_{n}\right) \\
& =d\left(y_{n}, T^{n} y_{n}\right)+d\left(T T^{n-1} y_{n}, T y_{n}\right) \\
& \leq d\left(y_{n}, T^{n} y_{n}\right)+L d\left(T^{n-1} y_{n}, y_{n}\right) \rightarrow 0 \quad(\text { as } n \rightarrow \infty) .
\end{aligned}
$$

Next, we will show that $\left\{y_{n}\right\}$ contains a subsequence converging strongly to $\tilde{x}$ such that $\tilde{x}=P_{F(T)} f(\tilde{x})$, which is equivalent to the following variational inequality:

$$
\langle\overrightarrow{\tilde{x} f(\tilde{x})}, \overrightarrow{x \tilde{x}}\rangle \geq 0, \quad \forall x \in F(T)
$$

Since $\left\{y_{n}\right\}$ is bounded, there exists a subsequence $\left\{y_{n_{j}}\right\}$ of $\left\{y_{n}\right\}$ which $\Delta$-converges to $\tilde{x}$. By Lemmas 2.4, 2.6, we may assume that $\left\{y_{n_{j}}\right\} \Delta$-converges to a point $\tilde{x}$ and $\tilde{x} \in F(T)$. It follows from Lemma 2.10 (i) that

$$
\begin{aligned}
& d^{2}\left(y_{n_{j}}, \tilde{x}\right)=\left\langle\overrightarrow{y_{n_{j}}} \overrightarrow{\tilde{x}}, \overrightarrow{y_{n_{j}}} \overrightarrow{\tilde{x}}\right\rangle
\end{aligned}
$$

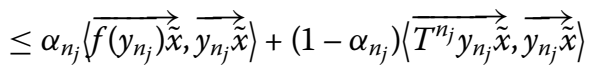

$$
\begin{aligned}
& \leq \alpha_{n_{j}}\left\langle\overrightarrow{f\left(y_{n_{j}}\right)} \overrightarrow{\tilde{x}}, \overrightarrow{y_{n_{j}}} \overrightarrow{\tilde{x}}\right\rangle+\left(1-\alpha_{n_{j}}\right) d\left(T^{n_{j}} y_{n_{j}}, \tilde{x}\right) d\left(y_{n_{j}}, \tilde{x}\right) \\
& \leq \alpha_{n_{j}}\left|\overrightarrow{f\left(y_{n_{j}}\right)} \vec{x}, \overrightarrow{y_{n_{j}}} \overrightarrow{\tilde{x}}\right\rangle+\left(1-\alpha_{n_{j}}\right) k_{n_{j}} d\left(y_{n_{j}}, \tilde{x}\right) d\left(y_{n_{j}}, \tilde{x}\right) \\
& =\alpha_{n_{j}}\left|\overrightarrow{f\left(y_{n_{j}}\right)} \vec{x}, \overrightarrow{y_{n_{j}}} \overrightarrow{\tilde{x}}\right\rangle+\left(1-\alpha_{n_{j}}\right) k_{n_{j}} d^{2}\left(y_{n_{j}}, \tilde{x}\right) \text {. }
\end{aligned}
$$

It follow that

$$
\begin{aligned}
& d^{2}\left(y_{n_{j}}, \tilde{x}\right) \leq \frac{\alpha_{n_{j}}}{\left(1-\left(1-\alpha_{n_{j}}\right) k_{n_{j}}\right)}\left\langle\overrightarrow{f\left(y_{n_{j}}\right) \vec{x}}, \overrightarrow{y_{n_{j}}} \vec{x}\right\rangle \\
& =\frac{\alpha_{n_{j}}}{\left(1-\left(1-\alpha_{n_{j}}\right) k_{n_{j}}\right)}\left[\left|\overrightarrow{f\left(y_{n_{j}}\right) f(\vec{x})}, \overrightarrow{y_{n_{j}}} \overrightarrow{\tilde{x}}\right\rangle+\left\langle\overrightarrow{f(\tilde{x})} \vec{x}, \overrightarrow{y_{n_{j}}} \vec{x}\right)\right]
\end{aligned}
$$




$$
\begin{aligned}
& \leq \frac{\alpha_{n_{j}}}{\left(1-\left(1-\alpha_{n_{j}}\right) k_{n_{j}}\right)}\left[d\left(f\left(y_{n_{j}}\right), f(\tilde{x})\right) d\left(y_{n_{j}}, \tilde{x}\right)+\left(\overrightarrow{f(\tilde{x}) \tilde{x},} \overrightarrow{y_{n_{j}} \tilde{x}}\right)\right] \\
& \leq \frac{\alpha_{n_{j}}}{\left(1-\left(1-\alpha_{n_{j}}\right) k_{n_{j}}\right)}\left[\alpha d^{2}\left(y_{n_{j}}, \tilde{x}\right)+\left(\overrightarrow{f(\tilde{x})} \overrightarrow{\tilde{x}}, \overrightarrow{y_{n_{j}}} \overrightarrow{\tilde{x}}\right)\right],
\end{aligned}
$$

and hence

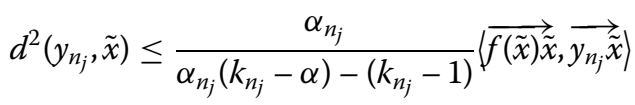

$$
\begin{aligned}
& \leq \frac{1}{1-\alpha}\left\langle\overrightarrow{f(\tilde{x})} \overrightarrow{\tilde{x}}, \overrightarrow{y_{n_{j}}} \overrightarrow{\tilde{x}}\right| \text {. }
\end{aligned}
$$

Since $\left\{y_{n_{j}}\right\} \Delta$-converges to $\tilde{x}$, by Lemma 2.7 , we have

$$
\limsup _{n \rightarrow \infty}\left\langle\overrightarrow{f(\tilde{x}) \tilde{x},} \overrightarrow{y_{n_{j}} \tilde{x}}\right\rangle \leq 0
$$

It follows from (17) that $\left\{y_{n_{j}}\right\}$ converges strongly to $\tilde{x}$. Next, we show that $\tilde{x}$ solves the variational inequality (15). Applying Lemma 2.3, for any $q \in F(T)$,

$$
\begin{aligned}
d^{2}\left(y_{n_{j}}, q\right) & =d^{2}\left(\alpha_{n_{j}} f\left(y_{n_{j}}\right) \oplus\left(1-\alpha_{n_{j}}\right) T^{n_{j}} y_{n_{j}}, q\right) \\
& \leq \alpha_{n_{j}} d^{2}\left(f\left(y_{n_{j}}\right), q\right)+\left(1-\alpha_{n_{j}}\right) d^{2}\left(T^{n_{j}} y_{n_{j}}, q\right)-\alpha_{n_{j}}\left(1-\alpha_{n_{j}}\right) d^{2}\left(f\left(y_{n_{j}}\right), T^{n_{j}} y_{n_{j}}\right) \\
& \leq \alpha_{n_{j}} d^{2}\left(f\left(y_{n_{j}}\right), q\right)+\left(1-\alpha_{n_{j}}\right) k_{n_{j}}^{2} d^{2}\left(y_{n_{j}}, q\right)-\alpha_{n_{j}}\left(1-\alpha_{n_{j}}\right) d^{2}\left(f\left(y_{n_{j}}\right), T^{n_{j}} y_{n_{j}}\right),
\end{aligned}
$$

and hence

$$
\left(1-\alpha_{n_{j}}\right) d^{2}\left(f\left(y_{n_{j}}\right), T^{n_{j}} y_{n_{j}}\right)+k_{n_{j}}^{2} d\left(y_{n_{j}}, q\right) \leq d^{2}\left(f\left(y_{n_{j}}\right), q\right)+\frac{k_{n_{j}}^{2}-1}{\alpha_{n_{j}}} d^{2}\left(y_{n_{j}}, q\right) .
$$

We then have

$$
\left(1-\alpha_{n_{j}}\right) d^{2}\left(f\left(y_{n_{j}}\right), T^{n_{j}} y_{n_{j}}\right)+k_{n_{j}}^{2} d\left(y_{n_{j}}, q\right) \leq d^{2}\left(f\left(y_{n_{j}}\right), q\right)+\frac{k_{n_{j}}-1}{\alpha_{n_{j}}} \bar{M},
$$

where $\bar{M}=\left(k_{n_{j}}+1\right) d^{2}\left(y_{n_{j}}, q\right)$. Since $y_{n_{j}} \rightarrow \tilde{x}$ and by (16), we have $T^{n_{j}} y_{n_{j}} \rightarrow \tilde{x}$. It follows from $\alpha_{n_{j}} \rightarrow 0, k_{n_{j}} \rightarrow 1, \frac{k_{n_{j}}-1}{\alpha_{n_{j}}} \rightarrow 0$, and continuity of the metric distance $d$ that

$$
d^{2}(f(\tilde{x}), \tilde{x})+d^{2}(\tilde{x}, q) \leq d^{2}(f(\tilde{x}), q) .
$$

Hence

$$
0 \leq \frac{1}{2}\left[d^{2}(\tilde{x}, \tilde{x})+d^{2}(f(\tilde{x}), q)-d^{2}(\tilde{x}, q)-d^{2}(f(\tilde{x}), \tilde{x})\right]=\langle\overrightarrow{\tilde{x} f(\tilde{x})}, \overrightarrow{q \tilde{x}}\rangle, \quad \forall q \in F(T) .
$$

That is, $\tilde{x}$ solves the inequality (15). Assume there exists a subsequence $\left\{y_{n_{k}}\right\}$ of $\left\{y_{n}\right\}$ which $\Delta$-converges to $\hat{x}$ by the same argument; we see that $\hat{x} \in F(T)$ and solves the variational inequality (15), i.e.,

$$
\langle\overrightarrow{\tilde{x} f(\tilde{x})}, \overrightarrow{\tilde{x} \hat{x}}\rangle \leq 0
$$


and

$$
\langle\overrightarrow{\hat{x} f(\hat{x}),}, \overrightarrow{\hat{x}} \tilde{x}\rangle \leq 0
$$

Adding up (19) and (20), we obtain

$$
\begin{aligned}
& 0 \geq\langle\overrightarrow{\tilde{x} f(\vec{x})}, \overrightarrow{\tilde{x} \hat{x}}\rangle-|\overrightarrow{\hat{x} f(\hat{x})}, \overrightarrow{\tilde{x} \hat{x}}| \\
& =\langle\overrightarrow{\tilde{x} f(\hat{x})}, \overrightarrow{\tilde{x} \hat{x}}\rangle+\langle\overrightarrow{f(\hat{x}) f(\vec{x})}, \overrightarrow{\tilde{x} \hat{x}}\rangle-\langle\overrightarrow{\hat{x} \tilde{x}}, \overrightarrow{\tilde{x} \hat{x}}\rangle-\langle\overrightarrow{\tilde{x} f(\hat{x})}, \overrightarrow{\tilde{x} \hat{x}}\rangle \\
& =\langle\overrightarrow{\tilde{x} \hat{x}}, \overrightarrow{\tilde{x} \hat{x}}\rangle-\langle\overrightarrow{f(\hat{x}) f(\vec{x})}, \vec{x} \vec{x}\rangle \\
& \geq\langle\overrightarrow{\tilde{x} \hat{x}}, \overrightarrow{\tilde{x} \hat{x}}\rangle-d(f(\hat{x}), f(\tilde{x})) d(\hat{x}, \tilde{x}) \\
& \geq d^{2}(\tilde{x}, \hat{x})-\alpha d(\hat{x}, \tilde{x}) d(\hat{x}, \tilde{x}) \\
& =d^{2}(\tilde{x}, \hat{x})-\alpha d^{2}(\hat{x}, \tilde{x}) \\
& =(1-\alpha) d^{2}(\tilde{x}, \hat{x}) .
\end{aligned}
$$

Since $0<\alpha<1$, we have $d(\tilde{x}, \hat{x})=0$, and so $\tilde{x}=\hat{x}$. Hence $y_{n}$ converges strongly as $n \rightarrow \infty$ to $\tilde{x}$, which solves the variational inequality (15).

Now, we present a strong convergence theorem for an asymptotically nonexpansive mapping.

Theorem 3.2 Let $C$ be a closed convex subset of a complete $C A T(0)$ space $X$, and let $T$ : $C \rightarrow C$ be an asymptotically nonexpansive mapping with a sequence $\left\{k_{n}\right\} \subset[1,+\infty)$ and $\lim _{n \rightarrow \infty} k_{n}=1$ such that $F(T) \neq \emptyset$. Let $f$ be a contraction on $C$ with coefficient $0<\alpha<1$. For the arbitrary initial point $x_{0} \in C$, let $\left\{x_{n}\right\}$ be generated by

$$
x_{n+1}=\alpha_{n} f\left(x_{n}\right) \oplus\left(1-\alpha_{n}\right) T^{n} x_{n}, \quad \forall n \geq 0,
$$

where $\left\{\alpha_{n}\right\} \subset(0,1)$ satisfies the following conditions:

(i) $\lim _{n \rightarrow \infty} \alpha_{n}=0$;

(ii) $\sum_{n=0}^{\infty} \alpha_{n}=\infty$;

(iii) $\lim _{n \rightarrow \infty} \frac{k_{n}-1}{\alpha_{n}}=0$;

(iv) $T$ satisfies the asymptotically regularity $\lim _{n \rightarrow \infty} d\left(x_{n}, T^{n} x_{n}\right)=0$.

Then $\left\{x_{n}\right\}$ converges strongly as $n \rightarrow \infty$ to $\tilde{x}$ such that $\tilde{x}=P_{F(T)} f(\tilde{x})$, which is equivalent to the variational inequality (15).

Proof We first show that the sequence $\left\{x_{n}\right\}$ is bounded. By condition (iv), for any $0<\varepsilon<$ $1-\alpha$ and sufficient large $n \geq 0$, we have $k_{n}-1 \leq \varepsilon \alpha_{n}$. For any $p \in F(T)$, we have

$$
\begin{aligned}
d\left(x_{n+1}, p\right) & =d\left(\alpha_{n} f\left(x_{n}\right) \oplus\left(1-\alpha_{n}\right) T^{n} x_{n}, p\right) \\
& \leq \alpha_{n} d\left(f\left(x_{n}\right), p\right)+\left(1-\alpha_{n}\right) d\left(T^{n} x_{n}, p\right) \\
& \leq \alpha_{n}\left(d\left(f\left(x_{n}\right), f(p)\right)+d(f(p), p)\right)+\left(1-\alpha_{n}\right) d\left(T^{n} x_{n}, p\right) \\
& \leq \alpha_{n} \alpha d\left(x_{n}, p\right)+\alpha_{n} d(f(p), p)+\left(1-\alpha_{n}\right) k_{n} d\left(x_{n}, p\right)
\end{aligned}
$$




$$
\begin{aligned}
& =\left(k_{n}\left(1-\alpha_{n}\right)+\alpha \alpha_{n}\right) d\left(x_{n}, p\right)+\alpha_{n} d(f(p), p) \\
& =\left(1+\left(k_{n}-1\right)-\left(k_{n}-\alpha\right) \alpha_{n}\right) d\left(x_{n}, p\right)+\alpha_{n} d(f(p), p) \\
& \leq\left(1+\varepsilon \alpha_{n}-\left(k_{n}-\alpha\right) \alpha_{n}\right) d\left(x_{n}, p\right)+\alpha_{n} d(f(p), p) \\
& =\left(1-\left(k_{n}-\alpha-\varepsilon\right) \alpha_{n}\right) d\left(x_{n}, p\right)+\alpha_{n} d(f(p), p) \\
& \leq\left(1-(1-\alpha-\varepsilon) \alpha_{n}\right) d\left(x_{n}, p\right)+\alpha_{n} d(f(p), p) \\
& \leq \max \left\{d\left(x_{n}, p\right), \frac{1}{(1-\alpha-\varepsilon)} d(f(p), p)\right\},
\end{aligned}
$$

for all $n \in \mathbb{N}$. This implies that $\left\{x_{n}\right\}$ is bounded, and so are $\left\{T^{n} x_{n}\right\}$ and $\left\{f\left(x_{n}\right)\right\}$. Next, we claim that $\lim _{n \rightarrow \infty} d\left(x_{n}, T x_{n}\right)=0$. Indeed we have

$$
\begin{aligned}
d\left(x_{n+1}, x_{n}\right) \leq & d\left(x_{n+1}, T^{n} x_{n}\right)+d\left(T^{n} x_{n}, x_{n}\right) \\
= & d\left(\alpha_{n} f\left(x_{n}\right) \oplus\left(1-\alpha_{n}\right) T^{n} x_{n}, T^{n} x_{n}\right)+d\left(T^{n} x_{n}, x_{n}\right) \\
\leq & \alpha_{n} d\left(f\left(x_{n}\right), T^{n} x_{n}\right)+d\left(T^{n} x_{n}, x_{n}\right) \rightarrow 0, \\
d\left(x_{n}, T^{n-1} x_{n}\right) & =d\left(\alpha_{n-1} f\left(x_{n-1}\right) \oplus\left(1-\alpha_{n-1}\right) T^{n-1} x_{n-1}, T^{n-1} x_{n}\right) \\
& \leq \alpha_{n-1} d\left(f\left(x_{n-1}\right), T^{n-1} x_{n}\right)+\left(1-\alpha_{n-1}\right) d\left(T^{n-1} x_{n-1}, T^{n-1} x_{n}\right) \\
& \leq \alpha_{n-1} d\left(f\left(x_{n-1}\right), T^{n-1} x_{n}\right)+\left(1-\alpha_{n-1}\right) k_{n-1} d\left(x_{n-1}, x_{n}\right) \rightarrow 0 .
\end{aligned}
$$

Therefore

$$
\begin{aligned}
d\left(x_{n}, T x_{n}\right) & \leq d\left(x_{n}, T^{n} x_{n}\right)+d\left(T^{n} x_{n}, T x_{n}\right) \\
& =d\left(x_{n}, T^{n} x_{n}\right)+k_{n} d\left(T^{n-1} x_{n}, x_{n}\right) \rightarrow 0 .
\end{aligned}
$$

By Theorem 3.1, we have

$$
\limsup _{n \rightarrow \infty}\left|\overrightarrow{f(\tilde{x}) \vec{x}, \overrightarrow{x_{n}} \vec{x}}\right\rangle \leq 0
$$

Finally, we prove that $x_{n} \rightarrow \tilde{x}$ as $n \rightarrow \infty$. For any $n \in \mathbb{N}$, we set $y_{n}=\alpha_{n} \tilde{x} \oplus\left(1-\alpha_{n}\right) T^{n} x_{n}$. It follows from Lemmas 2.9 and 2.10 (i), (ii) that

$$
\begin{aligned}
& d^{2}\left(x_{n+1}, \tilde{x}\right)=d^{2}\left(\alpha_{n} f\left(x_{n}\right) \oplus\left(1-\alpha_{n}\right) T^{n} x_{n}, \tilde{x}\right) \\
& \leq d^{2}\left(y_{n}, \tilde{x}\right)+2\left\langle\overrightarrow{x_{n+1} y_{n}}, \overrightarrow{x_{n+1}} \overrightarrow{\tilde{x}}\right\rangle \\
& \leq\left(\alpha_{n} d(\tilde{x}, \tilde{x})+\left(1-\alpha_{n}\right) d\left(T^{n} x_{n}, \tilde{x}\right)\right)^{2} \\
& \left.+2\left[\alpha_{n}\left|\overrightarrow{f\left(x_{n}\right) y_{n}}, \overrightarrow{x_{n+1} \tilde{x}}\right\rangle+\left(1-\alpha_{n}\right) \mid \overrightarrow{T^{n} x_{n} y_{n}}, \overrightarrow{x_{n+1}} \tilde{x}\right)\right] \\
& \leq\left(1-\alpha_{n}\right)^{2} k_{n}^{2} d^{2}\left(x_{n}, \tilde{x}\right)+2\left[\alpha_{n} \alpha_{n}\left\langle\overrightarrow{f\left(x_{n}\right) \tilde{x}}, \overrightarrow{x_{n+1}} \vec{x}\right\rangle\right. \\
& +\alpha_{n}\left(1-\alpha_{n}\right)\left|\overrightarrow{f\left(x_{n}\right) T^{n} x_{n}}, \overrightarrow{x_{n+1}} \overrightarrow{\tilde{x}}\right\rangle \\
& +\alpha_{n}\left(1-\alpha_{n}\right)\left\langle\overrightarrow{T^{n} x_{n} \tilde{x}}, \overrightarrow{x_{n+1}} \overrightarrow{\tilde{x}}\right\rangle \\
& +\left(1-\alpha_{n}\right)^{2}\left\langle\overrightarrow{T^{n} x_{n} T^{n} x_{n}}, \overrightarrow{x_{n+1} \tilde{x}}\right]
\end{aligned}
$$




$$
\begin{aligned}
& \leq\left(1-\alpha_{n}\right)^{2} k_{n}^{2} d^{2}\left(x_{n}, \tilde{x}\right)+2\left[\alpha_{n} \alpha_{n}\left\langle\overrightarrow{f\left(x_{n}\right)} \overrightarrow{\tilde{x}}, \overrightarrow{x_{n+1}} \overrightarrow{\tilde{x}}\right\rangle\right. \\
& +\alpha_{n}\left(1-\alpha_{n}\right)\left\langle\overrightarrow{f\left(x_{n}\right) T^{n} x_{n}}, \overrightarrow{x_{n+1} \tilde{x}}\right\rangle \\
& +\alpha_{n}\left(1-\alpha_{n}\right)\left\langle\overrightarrow{T^{n} x_{n} \tilde{x}}, \overrightarrow{x_{n+1}} \underset{\tilde{x}}{\longrightarrow}\right\rangle \\
& \left.+\left(1-\alpha_{n}\right)^{2} d\left(T^{n} x_{n}, T^{n} x_{n}\right) d\left(x_{n+1}, \tilde{x}\right)\right] \\
& =\left(1-\alpha_{n}\right)^{2} k_{n}^{2} d^{2}\left(x_{n}, \tilde{x}\right)+2\left[\alpha_{n} \alpha_{n}\left|\overrightarrow{f\left(x_{n}\right)} \vec{x}, \overrightarrow{x_{n+1}} \vec{x}\right\rangle\right. \\
& +\alpha_{n}\left(1-\alpha_{n}\right)\left|\overrightarrow{f\left(x_{n}\right) T^{n} x_{n}}, \overrightarrow{x_{n+1} \tilde{x}}\right\rangle \\
& \left.+\alpha_{n}\left(1-\alpha_{n}\right)\left\langle\overrightarrow{T^{n} x_{n} \tilde{x}}, \overrightarrow{x_{n+1}} \overrightarrow{\tilde{x}}\right\rangle\right] \\
& =\left(1-\alpha_{n}\right)^{2} k_{n}^{2} d^{2}\left(x_{n}, \tilde{x}\right)+2 \alpha_{n}\left|\overrightarrow{f\left(x_{n}\right)} \vec{x}, \overrightarrow{x_{n+1}} \vec{x}\right\rangle \\
& =\left(1-\alpha_{n}\right)^{2} k_{n}^{2} d^{2}\left(x_{n}, \tilde{x}\right)+2 \alpha_{n}\left\langle\overrightarrow{f\left(x_{n}\right) f(\tilde{x})}, \overrightarrow{x_{n+1}} \vec{x}\right\rangle \\
& +2 \alpha_{n}\left\langle\overrightarrow{f(\tilde{x}) \tilde{x}}, \overrightarrow{x_{n+1}} \vec{x}\right\rangle \\
& \leq\left(1-\alpha_{n}\right)^{2} k_{n}^{2} d^{2}\left(x_{n}, \tilde{x}\right)+2 \alpha_{n} \alpha d\left(x_{n}, \tilde{x}\right) d\left(x_{n+1}, \tilde{x}\right) \\
& +2 \alpha_{n}\left\langle\overrightarrow{f(\tilde{x}) \tilde{x}}, \overrightarrow{x_{n+1}} \vec{x}\right\rangle \\
& \leq\left(1-\alpha_{n}\right)^{2} k_{n}^{2} d^{2}\left(x_{n}, \tilde{x}\right)+\alpha_{n} \alpha\left(d^{2}\left(x_{n}, \tilde{x}\right)+d^{2}\left(x_{n+1}, \tilde{x}\right)\right) \\
& +2 \alpha_{n}\left\langle\overrightarrow{f(\tilde{x}) \tilde{x}}, \overrightarrow{x_{n+1}} \overrightarrow{\tilde{x}}\right\rangle \text {. }
\end{aligned}
$$

Since $\left\{\alpha_{n}\right\}$ and $\left\{x_{n}\right\}$ are bounded, there is $M>0$ such that

$$
\frac{1}{1-\alpha \alpha_{n}} k_{n}^{2} d^{2}\left(x_{n}, \tilde{x}\right) \leq M .
$$

It follows that

$$
\begin{aligned}
& d^{2}\left(x_{n+1}, \tilde{x}\right) \leq \frac{\left(1-\alpha_{n}\right)^{2} k_{n}^{2}+\alpha \alpha_{n}}{1-\alpha \alpha_{n}} d^{2}\left(x_{n}, \tilde{x}\right)+\frac{2 \alpha_{n}}{1-\alpha \alpha_{n}}\left\langle\overrightarrow{f(\tilde{x}) \tilde{x}}, \overrightarrow{x_{n+1}} \overrightarrow{\tilde{x}}\right\rangle \\
& \leq \frac{\left(1-2 \alpha_{n}\right) k_{n}^{2}+\alpha \alpha_{n}}{1-\alpha \alpha_{n}} d^{2}\left(x_{n}, \tilde{x}\right)+\frac{2 \alpha_{n}}{1-\alpha \alpha_{n}}\left\langle\overrightarrow{f(\tilde{x}) \tilde{x}}, \overrightarrow{x_{n+1}} \overrightarrow{\tilde{x}}\right\rangle+\alpha_{n}^{2} M \\
& \leq\left(1-\frac{1-2 \alpha \alpha_{n}-\left(1-2 \alpha_{n}\right) k_{n}^{2}}{1-\alpha \alpha_{n}}\right) d^{2}\left(x_{n}, \tilde{x}\right)+\frac{2 \alpha_{n}}{1-\alpha \alpha_{n}}\left\langle\overrightarrow{f(\tilde{x}) \tilde{x},} \overrightarrow{x_{n+1} \tilde{x}}\right\rangle+\alpha_{n}^{2} M \\
& \leq\left(1-\frac{1-2 \alpha \alpha_{n}-\left(1-2 \alpha_{n}\right) k_{n}^{2}}{1-\alpha \alpha_{n}}\right) d^{2}\left(x_{n}, \tilde{x}\right) \\
& +\alpha_{n}\left(\frac{2}{1-\alpha \alpha_{n}}\left\langle\overrightarrow{f(\tilde{x}) \tilde{x}}, \overrightarrow{x_{n+1}} \overrightarrow{\tilde{x}}\right\rangle+\alpha_{n}^{2} M\right) \text {. }
\end{aligned}
$$

Now, taking $\gamma_{n}=\frac{1-2 \alpha \alpha_{n}-\left(1-2 \alpha_{n}\right) k_{n}^{2}}{1-\alpha \alpha_{n}}, \delta_{n}=\alpha_{n}\left(\frac{2}{1-\alpha \alpha_{n}}\left\langle\overrightarrow{f(\tilde{x}) \tilde{x}}, \overrightarrow{x_{n+1}} \overrightarrow{\tilde{x}}\right\rangle+\alpha_{n}^{2} M\right)$; applying Lemma 2.8 and (22), we can conclude that $x_{n} \rightarrow \tilde{x}$.

If $T: C \rightarrow C$ in Theorem 3.2 is a nonexpansive mapping, we can obtain the following result immediately.

Corollary 3.3 [20, Theorem 3.4] Let $C$ be a closed convex subset of a complete CAT(0) space $X$, and let $T: C \rightarrow C$ be a nonexpansive mapping with $F(T) \neq \emptyset$. Let $f$ be a con- 
traction on $C$ with coefficient $0<\alpha<1$. For the arbitrary initial point $x_{0} \in C$, let $\left\{x_{n}\right\}$ be generated by

$$
x_{n+1}=\alpha_{n} f\left(x_{n}\right) \oplus\left(1-\alpha_{n}\right) T x_{n}, \quad \forall n \geq 0,
$$

where $\left\{\alpha_{n}\right\} \subset(0,1)$ satisfies the following conditions:

(i) $\lim _{n \rightarrow \infty} \alpha_{n}=0$;

(ii) $\sum_{n=0}^{\infty} \alpha_{n}=\infty$;

(iii) either $\sum_{n=0}^{\infty}\left|\alpha_{n+1}-\alpha_{n}\right|<\infty$ or $\lim _{n \rightarrow \infty}\left(\alpha_{n+1} / \alpha_{n}\right)=1$.

Then $\left\{x_{n}\right\}$ converges strongly as $n \rightarrow \infty$ to $\tilde{x}$ such that $\tilde{x}=P_{F(T)} f(\tilde{x})$ which is equivalent to the variational inequality (15).

\section{Competing interests}

The authors declare that they have no competing interests.

\section{Authors' contributions}

All authors read and approved the final manuscript.

\section{Author details}

${ }^{1}$ Department of Mathematics, Faculty of Science, Naresuan University, Phitsanulok, 65000, Thailand. ${ }^{2}$ Centre of Excellence in Mathematics, CHE, Si Ayutthaya Rd., Bangkok, 10400, Thailand. ${ }^{3}$ Program in Mathematics, Faculty of Education, Pibulsongkram Rajabhat University, Phitsanulok, 65000, Thailand.

\section{Acknowledgements}

This research is supported by the Centre of Excellence in Mathematics, the Commission on Higher Education, Thailand.

\section{Received: 30 August 2014 Accepted: 27 January 2015 Published online: 13 February 2015}

\section{References}

1. Bruhat, F, Tits, J: Groupes réductifs sur un corps local. I. Données radicielles valuées. Publ. Math. Inst. Hautes Études Sci. 41, 5-251 (1972)

2. Banach, S: Metric Spaces of Nonpositive Curvature. Springer, New York (1999)

3. Brown, KS: Building. Springer, New York (1989)

4. Goebel, K, Reich, S: Uniform Convexity, Hyperbolic Geometry, and Nonexpansive Mappings. Series of Monographs and Textbooks in Pure and Applied Mathematics, vol. 83. Dekker, New York (1984)

5. Berg, ID, Nikoleav, IG: Quasilinearization and curvature of Alexandrov spaces. Geom. Dedic. 133, 195-218 (2008)

6. Kakavandi, BA, Amini, M: Duality and subdifferential for convex functions on complete CAT(0) metric spaces. Nonlinear Anal. 73, 3450-3455 (2010)

7. Dehghan, H, Rooin, J: A characterization of metric projection in CAT(0) spaces. In: Proceedings of Interational Conference on Functional Equation, Geometric Functions and Applications (ICFGA 2012), Payame Noor University, Tabriz, Iran, 10-12 May 2012, pp. 41-43 (2012)

8. Kirk, WA: Geodesic geometry and fixed point theory. In: Seminar of Mathematical Analysis (Malaga/Seville, 2002/2003), pp. 195-225. Univ. Sevilla Secr. Publ., Seville (2003)

9. Bridson, M, Haefliger, A: Sur les opérations dans les ensembles abstraits et leur application aux équations intégrales. Fundam. Math. 3, 133-181 (1922)

10. Cho, YJ, Ćirić, L, Wang, SH: Convergence theorems for nonexpansive semigroups in CAT(0) spaces. Nonlinear Anal. 74, 6050-6059 (2011)

11. Dhompongsa, S, Panyanak, B: On $\Delta$-convergence theorems in CAT(0) spaces. Comput. Math. Appl. 56, 2572-2579 (2008)

12. Dhompongsa, S, Kirk, WA, Panyanak, B: Nonexpansive set-valued mappings in metric and Banach spaces. J. Nonlinear Convex Anal. 8, 33-45 (2007)

13. Dhompongsa, S, Kirk, WA, Sims, B: Fixed points of uniformly Lipschitzian mappings. Nonlinear Anal. 65, 762-772 (2006)

14. Shi, LY, Chen, RD: Strong convergence of viscosity approximation methods for nonexpansive mappings in CAT(0) spaces. J. Math. Appl. (2012). doi:10.1155/2012/421050

15. Saejung, S: Halpern's iteration in CAT(0) spaces. Fixed Point Theory Appl. (2010). doi:10.1155/2010/471781

16. Goebel, K, Kirk, WA: A fixed point theorem for asymptotically nonexpansive mappings. Proc. Am. Math. Soc. 35 , 171-174 (1972)

17. Browder, FE: Fixed point theorems for noncompact mappings in Hilbert spaces. Proc. Natl. Acad. Sci. USA 53, 1272-1276 (1965)

18. Reich, S: Strong convergence theorems for resolvents of accretive operators in Banach spaces. J. Math. Anal. Appl. 75, 287-292 (1980)

19. Halpern, B: Fixed points of nonexpansive maps. Bull. Am. Math. Soc. 73, 957-961 (1967) 
20. Wangkeeree, R, Preechasilp, P: Viscosity approximation methods for nonexpansive mappings in CAT(0) spaces. J. Inequal. Appl. 2013, Article ID 93 (2013)

21. Shi, LY, Chen, RD, Wu, YJ: $\Delta$-Convergence problems for asymptotically nonexpansive mappings in CAT(0) spaces. Abstr. Appl. Anal. 2013, Article ID 251705 (2013)

22. Wangkeeree, R, Preechasilp, P: Viscosity approximation methods for nonexpansive semigroups in CAT(0) spaces. Fixed Point Theory Appl. 2013, Article ID 160 (2013)

23. Reich, S, Shemen, L: A note on Halpern's algorithm in the Hilbert ball. J. Nonlinear Convex Anal. 14, 853-862 (2013)

24. Kopecka, K, Reich, S: Approximating fixed points in the Hilbert ball. J. Nonlinear Convex Anal. 15, 819-829 (2013)

25. Kumam, P, Saluja, GS, Nashine, HK: Convergence of modified S-iteration process for two asymptotically nonexpansive mappings in the intermediate sense in CAT(0) spaces. J. Inequal. Appl. 2014, Article ID 368 (2014)

26. Lim, TC: Remarks on some fixed point theorems. Proc. Am. Math. Soc. 60, 179-182 (1976)

27. Kirk, WA, Panyanak, B: A concept of convergence in geodesic spaces. Nonlinear Anal. 68, 3689-3696 (2008)

28. Hussain, N, Khamsi, MA: On asymptotic pointwise contraction in metric space. Nonlinear Anal., Theory Methods Appl. 71, 4423-4429 (2009)

29. Kakavandi, BA: Weak topologies in complete CAT(0) metric spaces. Proc. Am. Math. Soc. 141, 1029-1039 (2013)

30. Xu, HK: An iterative approach to quadratic optimization. J. Optim. Theory Appl. 116, 659-678 (2003)

\section{Submit your manuscript to a SpringerOpen ${ }^{\mathcal{O}}$ journal and benefit from:}

- Convenient online submission

- Rigorous peer review

- Immediate publication on acceptance

- Open access: articles freely available online

- High visibility within the field

- Retaining the copyright to your article 